\title{
Spectrin: Structure, function and disease
}

\author{
ZHANG Rui ${ }^{1}$, ZHANG ChenYu ${ }^{1}$, ZHAO Qi $^{2} \&$ LI DongHai ${ }^{*}$ \\ ${ }^{1}$ Jiangsu Engineering Research Center for microRNA Biology and Biotechnology, State Key Laboratory of Pharmaceutical Biotechnology, \\ School of Life Sciences, Nanjing University, Nanjing 210093, China; \\ ${ }^{2}$ Institute of Biomedicine and Biotechnology, Shenzhen Institutes of Advanced Technology, Chinese Academy of Sciences, \\ Shenzhen 518055, China
}

Received November 21, 2012; accepted March 20, 2013

\begin{abstract}
Spectrin is a large, cytoskeletal, and heterodimeric protein composed of modular structure of $\alpha$ and $\beta$ subunits, it typically contains 106 contiguous amino acid sequence motifs called "spectrin repeats". Spectrin is crucial for maintaining the stability and structure of the cell membrane and the shape of a cell. Moreover, it contributes to diverse cell functions such as cell adhesion, cell spreading, and the cell cycle. Mutations of spectrin lead to various human diseases such as hereditary hemolytic anemia, type 5 spinocerebellar ataxia, cancer, as well as others. This review focuses on recent advances in determining the structure and function of spectrin as well as its role in disease.
\end{abstract}

erythrocyte, spectrin, cell cycle, mass spectrometry, disease

Spectrin is a cytoskeletal protein that was first discovered in erythrocytes and is important for maintaining the stability, structure, and shape of the cell membrane. Spectrin was first isolated as a membrane-associated protein from an erythrocyte component devoid of hemoglobin called "ghosts" [1]. Spectrin forms a complex two-dimensional intracellular network by interacting with intracellular proteins such as actin, ankyrin, and adducin (Figure 1) [2,3]. This network maintains the flexibility and shape of the cell membrane. The inherent elasticity of spectrin is one of the main factors that maintains the elasticity of the erythrocytes in the circulatory system [4]. Mutated spectrin also causes some blood diseases such as hemolytic anemia. Spectrin also helps to maintain the Golgi apparatus and signal transduction pathways in many cell types [5].

There are two $\alpha$ and five $\beta$ subunits of human spectrin $(\alpha \mathrm{I}, \alpha \mathrm{II}, \beta \mathrm{I}, \beta \mathrm{II}, \beta \mathrm{III}, \beta \mathrm{IV}$, and $\beta \mathrm{V}$ ). The $\alpha \mathrm{I}$ and $\alpha \mathrm{II}-$ spectrin

*Corresponding author (email: donghaili@nju.edu.cn) subunits are encoded by SPTA1 and SPTAN1, respectively. SPTAl is expressed in erythroid cells. In contrast to SPTA1, the SPTANI encodes several $\alpha$ II-spectrin isoforms that are expressed in all nonerythroid cells [6,7]. SPTB, SPTBN1, $S P T B N 2$, and SPTBN4 encode $\beta \mathrm{I}-\beta \mathrm{IV}$ spectrins, respectively, and SPTBN5 encodes $\beta \mathrm{V}$ spectrin that is expressed at a low level in many tissues [8]. Three spectrin isoforms are present in invertebrate cells as follows: $\alpha, \beta-\mathrm{G}$, and $\beta-\mathrm{H}$. The $\alpha$ subunits are encoded by a gene similar to mammalian SPTAN1, and $\beta-\mathrm{G}$ and $\beta-\mathrm{H}$ correspond to mammalian $\beta \mathrm{II}$ and $\beta \mathrm{V}$, respectively [9]. The relationship between spectrin subunits and genes is displayed in Table 1.

Spectrin is a large heterodimeric protein with a modular structure typically composed of 106 contiguous amino acid sequence motifs called "spectrin repeats". The molecular weights of the $\alpha$ and $\beta$ subunits are 280 and $246 \mathrm{kD}$, respectively. Spectrin is a member of the F-actin-crosslinking protein superfamily. This superfamily contains only proteins composed of spectrin repeats such as dystrophin and 
Table 1 Spectrin genes and isoforms

\begin{tabular}{|c|c|c|c|}
\hline Species & Subunit & Gene & Chromosomal location \\
\hline \multirow{3}{*}{$\begin{array}{l}\text { Caenorhabditis } \\
\text { elegans }\end{array}$} & $\alpha$ & $S p c-1$ & $\mathrm{X}$ \\
\hline & $\beta-G$ & Unc-70/bgs-1 & $\mathrm{V}$ \\
\hline & $\beta-\mathrm{H}$ & sma-1 & $\mathrm{V}$ \\
\hline \multirow{3}{*}{$\begin{array}{c}\text { Drosophila } \\
\text { melanogaster }\end{array}$} & $\alpha$ & I(3)dre3 & 3L 62B4-62B4 \\
\hline & $\beta-G$ & $\beta$-Spec & X 16B10-16B12 \\
\hline & $\beta-\mathrm{H}$ & karst & 3L 63D2-63D2 \\
\hline \multirow{7}{*}{ Homo sapiens } & $\alpha I$ & SPTA1 & $1 \mathrm{q} 21$ \\
\hline & $\alpha \mathrm{II}$ & SPTAN1 & $9 \mathrm{q} 34.11$ \\
\hline & $\beta \mathrm{I}$ & $S P T B$ & $14 \mathrm{q} 22-\mathrm{q} 23.2$ \\
\hline & $\beta I I$ & SPTBN1 & $2 q 21$ \\
\hline & $\beta \mathrm{III}$ & SPTBN2 & $11 \mathrm{q} 13$ \\
\hline & $\beta \mathrm{IV}$ & SPTBN4 & $19 q 13.13$ \\
\hline & $\beta \mathrm{V}$ & SPTBN5 & $15 \mathrm{q} 21$ \\
\hline
\end{tabular}

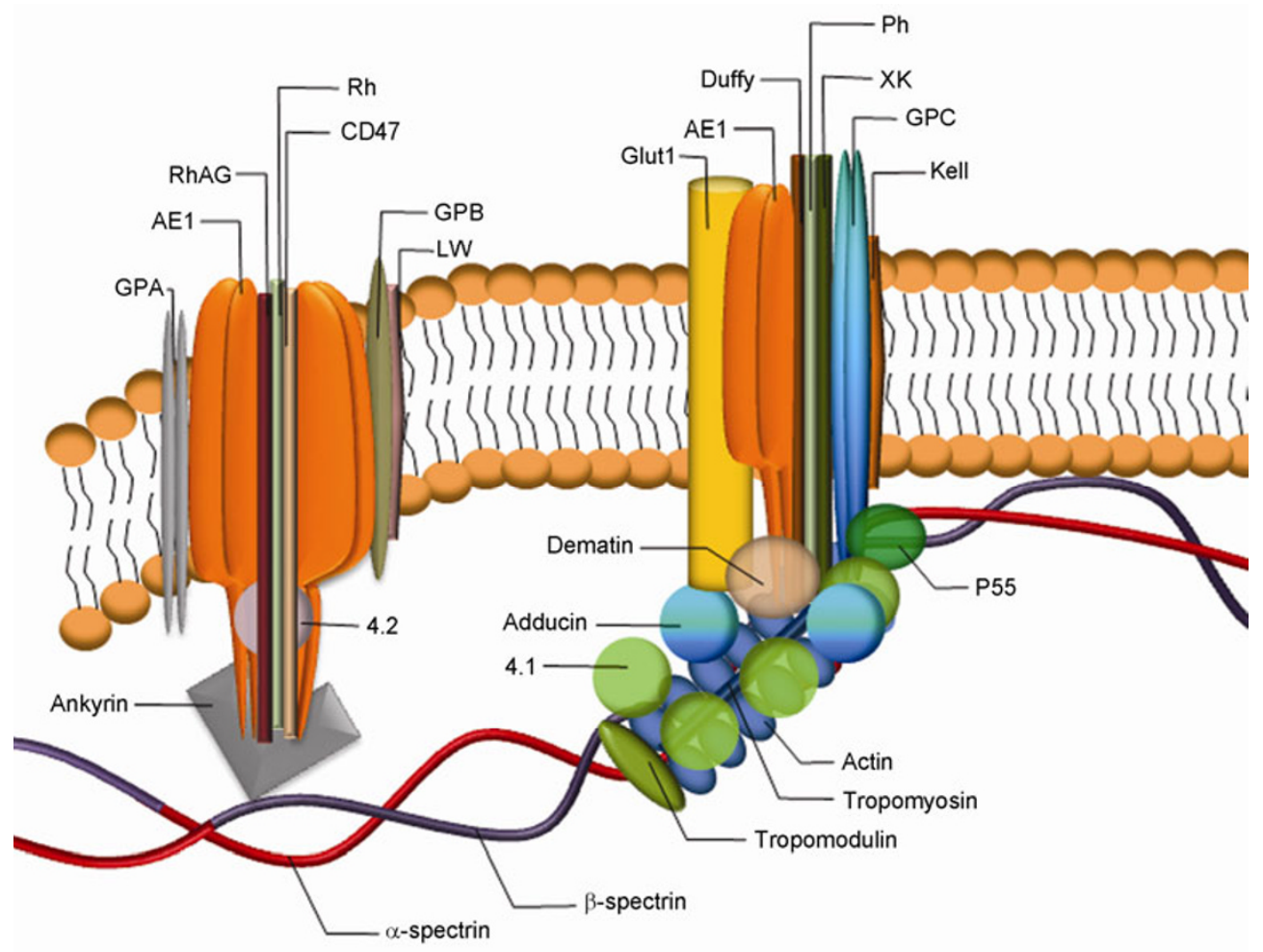

Figure 1 The spectrin-based skeleton model. Spectrin mainly interacts with actin, ankyrin and lipids, while establishing a complex intracellular two-dimensional network where the spectrin-actin interaction also requires accessory proteins such as tropomyosin, adducin, and dematin. These proteins help to maintain the stability of the spectrin-actin complex and participate in transmembrane protein interactions such as the interaction between Glut 1 and AE1. Ankyrin mediates the interactions between spectrin and other transmembrane proteins, such as AE1 and the Rh complex fixed on the spectrin-based skeleton through their interactions with ankyrin. AE1, anion exchanger; GPA, glycophorin A; GPB, glycophorin B; GPC, glycophorin C; Glut 1, glucose transporter 1; Rh, rhesus factor; RhAG, Rh-associated glycoprotein.

actin. These repeats might have been generated by gene duplication [10]. Spectrin repeats form a triple-helix supercoil connected by an $\alpha$ helical linker, thus allowing spectrin to expand and contract [11]. Furthermore, spectrin comprises numerous structural motifs, including actin-binding, pleckstrin homology, and Src homology 3 domains that contribute to spectrin function. These structural characteristics allow spectrin to participate in the important physiolog- ical processes described above.

\section{The structure of spectrin}

The amino acid sequences of spectrin $\alpha$ and $\beta$ subunits share approximately $30 \%$ similarity, and the subunits are associate side-to-side. Advanced research approaches reveal 
that the $\alpha$ and $\beta$ subunits interact primarily through hydrophobic bonds supported by electrostatic attraction [12]. Two anti-parallel subunits form a flexible 100-nm-long rod-like structure. These heterodimers are connected head to head, forming a 200-nm long tetramer. X-ray diffraction analysis of chicken-brain spectrin repeats reveals that each is composed of a triple helix bundle (A, B, and C). The triple helix repeats form a left-handed coiled-coil that is separated by a loop-and-turn region to form the complete spectrin molecule. These characteristics also make spectrin flexible [13]. The X-ray crystallographic data for the chicken-brain $\alpha$ spectrin triple $\alpha$-helix repeat bundle indicate that the connections between spectrin repeats can bend independently without affecting the adjacent connection [14].

Recently, our group determined the medium resolution three-dimensional structures of a spectrin dimer initiation site and a tetrameric head-head interaction site, using homology modeling and chemical cross-linking combined with mass spectrometry [15-18]. These findings led us to propose a spectrin dimer formation model called the zipper model. In this model, dimer starts to form at the spectrin dimerization initiation sites for the $\alpha$ and $\beta$ subunits ( $\alpha 20-21$ repeats with $\beta 1-2$ repeats) through electrostatic interactions. Other repeats then form an anti-parallel spectrin heterodimer and zips closed through hydrophobic interactions and electrostatic attraction. Finally, the N-terminus of the $\alpha$ subunit bends to the rear and interacts with the C-terminus of the $\beta$ subunits to form a "hairpin" configuration. To explain the structure and function of spectrin, we constructed a miniature spectrin that simulates the structural and functional properties of whole spectrin dimers and tetramers (Figure 2). The results generated from highperformance liquid chromatography initially provided nonimage data for spectrin "open" and "closed" dimers; chemical cross-linking combined with mass spectrometry results confirmed this structure. We then constructed a medium- resolution tetrameric structure containing seven spectrin repeats, indicating that the $\mathrm{AB}$ and $\mathrm{AC}$ surfaces of the helix bundle are important during inter-chain interactions [15-18].

The function of spectrin is closely related to its structure. Although these structures are very similar, their functional sites are different. These differences make non-erythrocytic spectrin more stable and erythrocyte spectrin more flexible. Except for the resolved spectrin repeat fragments, the crystal and NMR structures of the domains interacting with other proteins have also been resolved, including actin-binding [19] and pleckstrin homology domains [20] (Table 2).

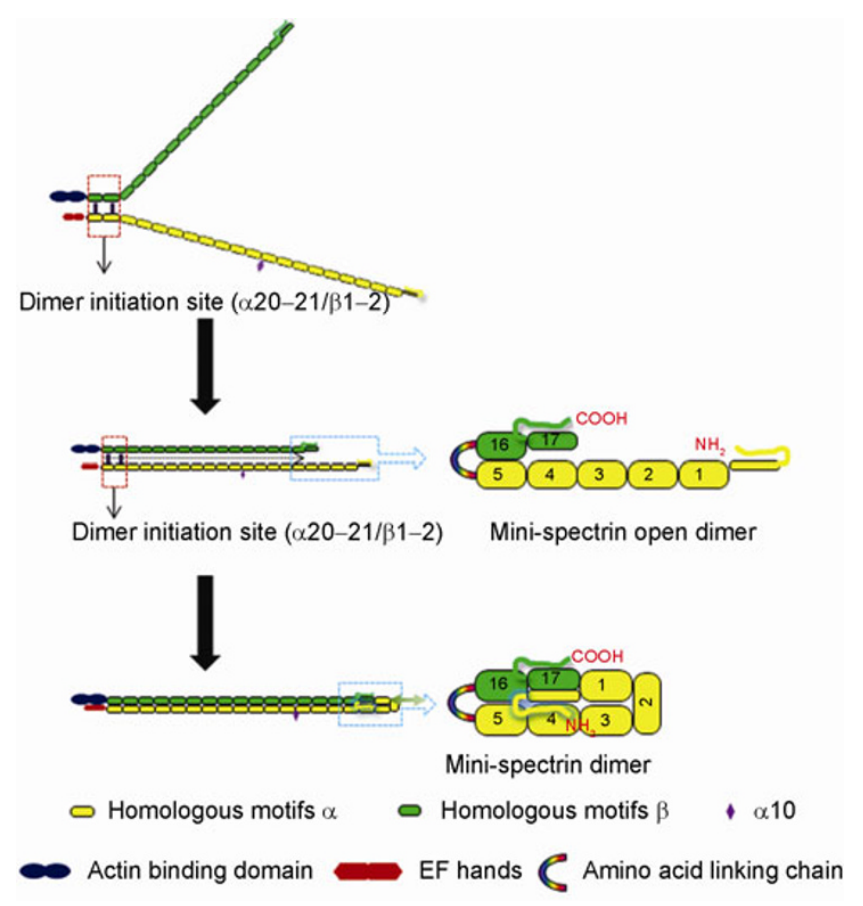

Figure 2 Mechanism of the 3-step "zipper" model of the spectrin heterodimer.

Table 2 High-resolution three-dimensional spectrin structures

\begin{tabular}{ccccc}
\hline PDBID & Subunit & Source & Content & Method \\
\hline 1 U06 & $\alpha$ & Chicken brain & SH3 domain & X-RAY \\
1 U4Q & $\alpha$ & Chicken brain & Repeats 15, 16, 17 & X-RAY \\
1 U5P & $\alpha$ & Chicken brain & Repeats 15, 16 & X-RAY \\
1 AJ3 & $\alpha$ & Chicken brain & Repeat 16 & SOLUTION NMR \\
1 CUN & $\alpha$ & Chicken brain & Tetramerization sites 16,17 & X-RAY \\
3LBX & $\alpha \beta$ & Human erythrocyte & Repeats 14, 15 & X-RAY \\
3KBT & $\beta$ & Human erythrocyte & N-terminal & X-RAY \\
3F31 & $\alpha$ & Chicken brain & Repeats 8,9 & X-RAY \\
1 S35 & $\beta$ & Human erythrocyte & N-terminal & X-RAY \\
1OWA & $\alpha$ & Human erythrocyte & SOLUTION NMR \\
1BKR & $\beta$ & Human erythrocyte & Calmodulin homology domain & X-RAY \\
1MPH & $\beta$ & Mouse & Pleckstrin homology domain & SOLUTION NMR \\
2SPC & & Sposophila & Spectrin repeat 14 & X-RAY \\
\hline
\end{tabular}




\section{The function of spectrin}

\subsection{Spectrin-based functional complex}

Spectrin is multifunctional, and spectrin-based networks are important for maintaining the shape and mechanical properties of erythrocytes. Clinical tests reveal that mutant forms of spectrin in erythrocytes make the cells more fragile, reduce their flexibility, and cause hereditary hemolytic anemia. Similarly, spectrin is required to maintain membrane stability and cell shape in nucleated cells [21,22]. Interactions between spectrin and ankyrin are vital for maintaining cell membrane stability. The structure of the ankyrinbinding domain of spectrin has been solved. It reveals that repeats $13-15$ of the spectrin $\beta$ subunit interact with ankyrin ZU5 domains through hydrophobic interactions and electrostatic attraction. Also, the interactions are involved in cell-cell interactions [23] and cellular signal transduction. Devarajan et al. [24] found that a spectrin-ankyrin-based skeleton may contribute to $\mathrm{Na}^{+} / \mathrm{K}^{+}$-ATPase transport from the endoplasmic reticulum to the Golgi apparatus. A vesicular spectrin-ankyrin adaptor protein trafficking system may mediate the capture and transport of many membrane proteins while cooperating with vesicular target molecules to transport cargo proteins. Recently, Cairo et al. [25] used bioinformatics methods to establish a model of the CD45-cytoskeleton interaction and elucidated that the spectrin-ankyrin-actin network significantly controls immunoreceptor mobility.

The N-terminal region of the spectrin $\beta$ subunit consists of two calponin homology domains $(\mathrm{CH} 1, \mathrm{CH} 2)$ that are mainly involved in binding protein 4.1R and actin [26]. Spectrin combines with F-actin and protein 4.1R to form the cross-cytoskeletal network of erythrocytes. The spectrin $\beta$ subunit interacts with a $10-\mathrm{kD}$ spectrin-actin-binding (SAB) domain of protein $4.1 \mathrm{R}$ through its $\mathrm{CH}$ domains. Spectrin has two actin-binding domains and two 4.1R-binding domains that are all located in two $\mathrm{CH}$ domains. The $\mathrm{N}$-terminal region of spectrin interacts with $4.1 \mathrm{R}$ and is regulated by phosphatidylinositol-4,5-bisphosphate (PIP2) $[27,28]$. Introducing a mutation that changes Trp-202 to Arg in spectrin can cause hereditary spherocytosis (HS) that alters the spectrin-4.1R interaction [29,30]. By interacting with various membrane proteins (Figure 3 ), spectrin provides a versatile platform for other proteins, making it a multi-functional protein.

\subsection{Spectrin-dependent cell adhesion and spreading}

Deletion of spectrin coding sequences seriously impairs cell adhesion and spreading, although few studies are available. A study on Drosophila shows that $\beta-\mathrm{H}$ spectrin interacts with proteins involved in morphogenesis that affects cell adhesion and migration [31]. The $\alpha$ II isoform interacts with the intrinsic protein $\beta 3$ that appears during early cell spreading and subsequently disappears [32]. Another study focuses on the spectrin $\beta$ subunit SH3-homology domain. Spectrins with SH3 domains may participate in activation of Rac and Rho GTPase activities [33]. Thus, overexpressing the spectrin SH3 domain protein may inhibit Rac and actin fiber formation, thus affecting cell stretching. Collec et al. [34] concluded that the stress fiber formation during cell spreading on laminin $511 / 521$ is influenced by the interaction between Lutheran/basal cell-adhesion molecule (Lu/ BCAM) and $\alpha$ II-spectrin. Moreover, spectrin serves as a signal relay between laminin 511/521 and actin during actin reorganization. Vasodilator-stimulated phosphoprotein (VASP) [35] and Ena/VASP-like protein (EVL) [36], which are members of their namesake Ena/VASP family, play a key role during actin remodeling and pseudopodia extension during the activation of immune cells. In the heart, Ena/ VASP and $\alpha$ II-spectrin assemble cardiac multiprotein complexes that regulate cytoplasmic actin networks [37]. These studies suggest that spectrin is crucial for cell adhesion and spreading.

\subsection{Spectrin and the cell cycle}

Spectrin is involved in the tumor growth factor (TGF)$\beta / \mathrm{SMAD}$ signaling pathway as a SMAD3/4 adaptor. The link between spectrin and the (TGF)- $\beta /$ SMAD signaling pathway was first indicated by the molecular cloning of a cDNA (elf1) encoding a $\beta$ spectrin homolog designated embryonic liver fodrin 1 (ELF1) [38]. ELF1 encodes a 220amino acid residue protein with an $\mathrm{N}$-terminal actin-binding domain. In situ hybridization studies revealed elfl expression in day 10 embryonic heart and day 11-11.5 hepatic tissues, suggesting that elf1 participates in the emergence of hepatocyte polarity during liver development [38]. Subtractive hybridization experiments using early embryonic mouse cDNA libraries prepared from the liver led to the isolation of three isoforms of a novel $\beta$ spectrin ELF, the longest of which was called elf3. The ELF3 cDNA sequence comprises 8172 nucleotides (nt) with an open reading frame (ORF) of $6465 \mathrm{nt}$ (nt 333-6797). A potential cryptic poly(A)+ (AATAA) signal is present at nt 7359 followed by a typical poly(A)+ tail at nt 8172 . The predicted elf3 sequence reveals that elf 3 encodes 2154 amino acid residues with a calculated isoelectric point of 5.36, with $81.6 \%$ overall similarity to that of human $\beta$ spectrin isoforms. Like other spectrins, ELF3 has an actin-binding domain but lacks a $\mathrm{PH}$ domain [39].

In mouse $e l f^{-/}$mutants, disrupting the adaptor protein ELF inhibits TGF- $\beta$ signaling through SMADs. Tang et al. [40] generated ELF-deficient mice using gene targeting. They were not able to generate homozygous mutant elf $^{-/-}$ mice, indicating that the elf mutation is a lethal recessive embryonic trait. Subsequently, they obtained degenerating 


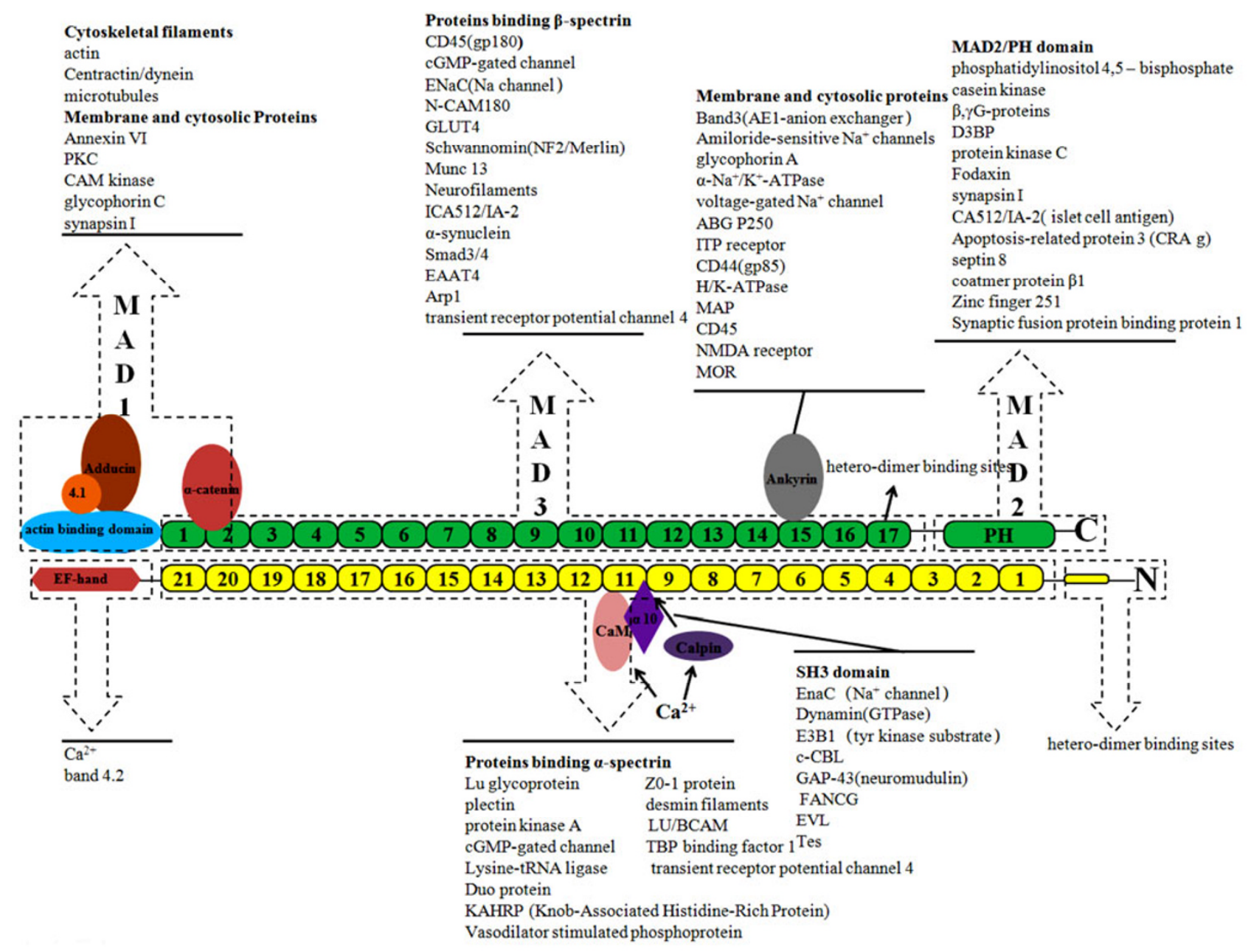

Figure 3 The interaction between spectrin and other proteins. Spectrin forms a reverse parallel heterodimer. $\beta$ I spectrin and $\beta$ II spectrin interact with the Golgi apparatus through their MAD1 and MAD2 domains, respectively. Repeats 13-15 of the spectrin $\beta$ subunit interact with ankyrin ZU5 domains through hydrophobic interactions and electrostatic attraction. The spectrin $\alpha$ subunit has a SH3 domain and is related to cell adhesion and stretching.

embryos from embryonic day 8.5 (E8.5) to E16.5. The elf ${ }^{-1}$ embryos were readily distinguished from their wild-type littermates by their smaller body and head sizes, as well as their lack of a branching network of vessels in the yolk sac at E9.5. At E11.5, the elf ${ }^{--}$embryos were severely distorted, exhibiting growth retardation and multiple defects. Elf ${ }^{-1-}$ mice display a phenotype similar to that of $\mathrm{Smad2}^{+/-}$, $\mathrm{Smad3}^{+/-}$mutant mice, including abnormal primary brain vesicle anatomy, craniofacial abnormalities, aberrant gut formation, severe hypoplasia of the liver, and distorted liver architecture. This similarity suggests that the ELF interacts with SMADs. Immunoprecipitation proved that TGF- $\beta$ triggers phosphorylation, associating ELF with SMAD3 and SMAD4. TGF- $\beta$ signaling could be rescued by transfecting elf ${ }^{--}$fibroblasts with the ELF COOH-terminal domain, which includes the ankyrin binding region, the phosphorylation sites at serine residues, and a hinge region regulating oligomer formation. However, it was not rescued by expression of SMAD3 or the ELF NH2-terminal domain.
Loss of the cooperative function of SMAD3 with ELF was discovered in primary biliary cirrhosis (PBC), autoimmune hepatitis $\mathrm{C}$, and chronic viral hepatitis. During the early stages of PBC, ELF expression and distribution increase in the cytoplasm and decrease in the cell membrane, and in its later stages, the ELF becomes highly overexpressed in the cytoplasm. In mouse elf $f^{-1}$ mutants, loss of ELF function causes $\mathrm{T}$ lymphocytic proliferation and the absence of intrahepatic bile ducts. The mislocalization of ELF was observed in $\mathrm{Smad}^{+-} / \mathrm{Smad}^{+/-}$mutants. These data suggest that ELF and SMAD3 are involved in the pathogenesis of PBC [41]. Interestingly, an exacerbated phenotype of early gastric hyperplasia, ectasia, foveolar gland dysplasia, and hamartomas with obstructing tumors at the antrum and pylorus were developed in $90 \%$ of the $\mathrm{elf}^{+1-} / \mathrm{Smad4}^{+/-}$heterozygous mutants. Abnormal mitosis, apoptosis, and glandular dilatation are observed in the polyps and hamartomas, suggesting a cooperative interaction between ELF and SMAD4 that enhances tumorigenesis [42]. 


\subsection{Spectrin and intracellular traffic}

Spectrin interacts with the Golgi apparatus to affect cell secretion. Moreover, the N-terminal domain of $\beta I$ spectrin contains a Golgi apparatus-binding site that includes actin-binding and MAD1 domains [24]. The MAD2 domain is present in some spectrins, which includes a PH domain. The MAD2 sequence in different spectrins varies widely, explaining its functional specificity [43]. In vitro experiments reveal that this domain binds to Golgi preparations, which is regulated by ADP ribosylation factor (ARF) that is a key molecule during Golgi apparatus dynamics [44]. The Golgi spectrin skeleton is also implicated in protein transport from the ER to the Golgi. The inhibitors of spectrin binding to Golgi stop the transport of vesicular stomatitis virus (VSV) $\mathrm{G}$ proteins from the endoplasmic reticulum to Golgi apparatus in semi-intact VSV-infected normal rat kidney (NRK) cells [44]. Using a spectrin $\beta$ III subunit containing the green fluorescent protein tag in transfected COS cells, spectrin decorated the tubular-vesicular transport intermediates moving along microtubules between the endoplasmic reticulum and the Golgi apparatus, requiring the participation of the MAD1 domain [45]. Salcedo-Sicilia et al. [46] suggested that $\beta$ III spectrin helps to maintain the structural integrity and secretory activity of the Golgi complex by associating with phosphatidylinositol 4-phosphate (PI4P).

\subsection{Spectrin and DNA repair}

Sridharan et al. [47] demonstrated that spectrin is important during the repair of DNA interstrand cross-links. The DNA repair proteins Fanconi anemia, complementation group A (FANCA), and excision repair cross-complementing rodent repair deficiency, complementation group 4 (ERCC4), as well as $\alpha$ II spectrin move to nuclear foci together after normal human cells are damaged by a DNA interstrand cross-linking agent (8-methox-ypsoralen) plus ultraviolet A (UVA) light. Cells from patients with Fanconi anemia that have decreased $\alpha$ II spectrin levels exhibit a decreased ability to repair DNA interstrand cross-links. Inhibiting the expression of $\alpha$ II spectrin using siRNA demonstrated that depleting the $\alpha$ II spectrin in normal cells increases chromosomal instability as ascertained by the increase in chromosomal aberrations/breaks [48]. Telomere maintenance after DNA interstrand cross-linking damage requires $\alpha$ II spectrin, which localizes to telomeres during $\mathrm{S}$ phase after DNA interstrand cross-linking damage and enhances the association with TRF1 and TRF2. EERC4 is a cross-linking repair protein that is recruited by $\alpha$ II spectrin to sites of damage-induced foci at the telomeres [49]. The relationships of spectrin and cell signaling of Caenorhabditis elegans and Drosophila melanogaster are summarized in Table 3; the role of spectrin in human cell signaling is summarized by Machnicka et al. [50].

\subsection{Spectrin and lipids}

Spectrin repeats interact with lipids [51]. Both $\alpha$ and $\beta$ spectrin have phosphatidylserine (PS) binding sites that interact with ankyrin and 4.1R. Manno et al. [52] discovered that asymmetric PS is important for maintaining the mechanical properties of cell membranes. Spectrin can bind to PS and combine with areas enriched in phosphatidylethanolamine (PE) [53]. The PE binding sites are located in repeats 14 and 15 of $\beta$ spectrin, containing 38 amino acid residues in the N-terminal domain with ankyrin binding sites. Ankyrin can inhibit the association of spectrin with PE [54]. Analyzing the spectrin-PE interaction sites using a series of point mutations revealed that W1771, L1775, M1778, and W1779 of $\beta$ spectrin are directly involved in binding to lipids [55]. Because PE and PS are asymmetrically distribut-

Table 3 The role of spectrin in cell signaling

\begin{tabular}{|c|c|c|}
\hline Species & Subunit & Function \\
\hline \multirow{3}{*}{$\begin{array}{l}\text { Caenorhabditis } \\
\text { elegans }\end{array}$} & $\alpha$ & $\begin{array}{l}\text { Body morphogenesis and growth } \\
\text { Larval morphogenesis } \\
\text { Regulation of locomotion }\end{array}$ \\
\hline & $\beta-G$ & $\begin{array}{l}\text { Regulation of growth rate, body morphogenesis and axon guidance } \\
\text { Neuron development }\end{array}$ \\
\hline & $\beta-\mathrm{H}$ & $\begin{array}{l}\text { Body morphogenesis and growth } \\
\text { Involved in the formation of collagen } \\
\text { Regulate organ growth }\end{array}$ \\
\hline \multirow{5}{*}{$\begin{array}{l}\text { Drosophila } \\
\text { melanogaster }\end{array}$} & $\alpha$ & $\begin{array}{l}\text { Formation cytoskeleton and maintain cell stability } \\
\text { Larval morphogenesis }\end{array}$ \\
\hline & $\beta-G$ & $\begin{array}{l}\text { Maintain the shape of the cell membrane } \\
\text { Axon midline choice point recognition }\end{array}$ \\
\hline & \multirow[b]{3}{*}{$\beta-H$} & $\begin{array}{l}\text { Nervous system establishment } \\
\text { Cell differentiation }\end{array}$ \\
\hline & & Endocytosis \\
\hline & & Maintain the stability of the cell membrane \\
\hline
\end{tabular}


ed on the cell membrane and the membrane in erythrocytes contains more PE, the ankyrin-based interactions between $\mathrm{PE}$ and spectrin in physiological processes are now recognized to be important.

\section{The role of spectrin in disease}

One of the most important functions of spectrin is to maintain cytoskeletal stability and mechanical properties; the absence of cytoskeletal components can generate weak and fragile cells that manifest clinically as types of hemolytic anemia, including hereditary elliptocytosis (HE) and HS. All HE cases involve defects in the spectrin-actin-4.1R complex. Mutations in SPTA1, SPTB, and 4.1R can cause $\mathrm{HE}$, and most are located in the tetramerization sites of spectrin $\alpha$ subunits that are the helical $C^{\prime}$ of repeats $\alpha 0$ or $\beta 17$. Some missense mutations reside in the first six repeats of the spectrin $\alpha \mathrm{I}$ subunit, and the $\mathrm{C}$-terminally truncated $\beta$ subunit (CpG islands) is a mutational hotspot [56]. The L260P mutation in $\alpha$ spectrin increases the stability of the closed dimer state, perturbing erythrocyte membranes with a fully functional mini-spectrin [57]. All HS have one common feature: the loss of cell membrane surface area causes a spherical phenotype and an increased level of erythrocyte osmotic fragility. Spectrin cannot be anchored to the cell membrane, and the destruction of membrane protein interactions such as that between spectrin and ankyrin, ankyrin and band3, band 3 and protein 4.2 , is the main mechanism that causes HS [58].

Spectrin represents $2 \%-3 \%$ of the total protein content of the brain and is located in neuronal synapses related to the connection between neurons in the presynaptic membrane and synaptic vesicles. Spectrin may interact with synaptic vesicle-specific protein MUNC13-1 [59]. The spectrin $\alpha$ II subunit is important for synaptic transmission and participates in the adhesion of synaptic vesicles in nerve endings. $\alpha$ and $\beta$ spectrin deletion mutations in Drosophila cause serious damage to neurotransmission and disrupt the subcellular localization of numerous synaptic proteins [60]. Therefore, spectrin might be involved in the localization of synaptic proteins, cysteine string protein (CSP) and disc-large (DLG) in neurons [56]. Knocking down the expression of $\beta G$ spectrin gene in $C$. elegans causes axonal growth defects, and an alteration in the structure of $\beta$ spectrin might facilitate amyloid $\beta$ deposition that causes Alzheimer's disease (AD) [61]. SPTBN2 mutations lead to spinocerebellar ataxia type 5. Abraham Lincoln's grandfather suffered from this disease, which is caused by the deletion of 13 amino acid residues from $\beta$ spectrin. The spectrin $\beta$ III subunit is mainly expressed in Purkinje cells. In these cells, the spectrin $\beta$ III subunit is mainly used to maintain the stability of membrane proteins. Mutations of spectrin $\beta$ subunit prevent proper positioning of EAAT4 and GluRg2 on the cell membrane. The absence of EAAT4 and GluRg2 leads to glutamate signaling abnormalities and spinocerebellar ataxia type 5 [62].

HE patients have a certain resistance to the parasite Plasmodium falciparum ( $P$. falciparum), and the absence of protein 4.1 and mutations in the spectrin self-association site can inhibit binding between the parasite and the host cell [63]. Most parasites combine with the erythrocyte skeleton through proteins before spreading on the cytoskeleton [64]. Parasite antigen binds protein 4.1 and moves through the erythrocyte membrane proteins. $P$. falciparum Knobassociated histidine-rich protein (KAHRP) binds the spectrin $\alpha 4$ repeat specifically before interacting with the erythrocyte skeleton [65]. Finally KAHRP combines with $P$. falciparum erythrocyte membrane protein 1 , which adheres to the parasitized erythrocyte cell on the vascular endothelium [66].

Spectrin may also provide host binding sites for enterohemorrhagic Escherichia coli (EHEC) that causes gastrointestinal disease. Ruetz et al. [67] used immunofluorescent microscopy to demonstrate that spectrin is recruited to EHEC attachment sites. The results on knockdown of spectrin by siRNA revealed that spectrin is necessary for efficient docking of the EHEC to host cells and recruiting IRSp53 to the pedestal, which is required for pedestal formation. These findings reveal that the spectrin cytoskeleton is part of the major host cell cytoskeletal network involved in events critical for pathogenesis by EHEC.

Visceral leishmaniasis (VL) is caused by Leishmania, and patients often suffer from anemia and other complications. Erythrocyte spectrin derived from patients suffering from this disease is glycosylated. Samanta et al. [68] isolated eight types of 9-O-acetylated sialoglycoproteins from the erythrocytes of VL patients. Mass spectrometric analyses revealed the presence of $\alpha$ - and $\beta$-spectrin. Compared with healthy persons, the spectrin in the patients with VL is highly sialylated. These sialylated spectrins may cause distortion and damage to the erythrocyte membranes. Other studies show that antibodies against 9-O-acetylated sialic acid glycoprotein increase in VL patients and activate calpain I and spectrin proteolysis to increase cellular oxidative stress and lipid peroxidation; therefore, the membrane becomes more fragile, explaining why patients with VL experience hemolysis and anemia [69].

Recent studies reveal that $\beta \mathrm{V}$ spectrin is involved in Usher syndrome type IB (USH1B) that is characterized by deafness and blindness. Spectrin $\beta \mathrm{V}$ homodimers are associated with USH1 proteins, opsin, and other phototransduction proteins that are transported toward the photoreceptor outer disks [70]. Also, spectrin serves as a biomarker for detecting several brain related injuries. Spectrin is a sub- 
strate for caspase-3 and calpain in lymphocytes, hematopoietic cells, and neurons during apoptosis. Caspase-3 and calpain cleave spectrin to form two degradation products (SBDPS), SBDP150 and SBDP120 that are named according to their relative electrophoretic mobilities [71]. Caspase-8 might cleave $\beta$-spectrin at its $\mathrm{N}$-terminal actin-binding domain, depending on the presence of protein 4.1 during fludarabine/mitoxantrone/dexamethasone-induced (FNDinduced) apoptosis [72].

\section{Conclusion and future prospects}

The structure of different subunits of spectrin has been identified; however, technical difficulties have prevented the determination of the three-dimensional protein structure at high resolution. So far, the largest spectrin structure is a medium-resolution structure of a seven-repeat bivalent tetramer produced using homology modeling coupled with chemical cross-linking and mass spectrometry. This complicated approach has the potential for determining the three-dimensional structure of full-length spectrin in the near future. Identification of spectrin mutations in various diseases contributes fruitful information to the understanding of spectrin functions in erythrocyte and non-erythrocyte. These results indicate an involvement of spectrins in a number of cell processes: cell cycle, cell adhesion, cell spreading, DNA repair and intracellular traffic. Although an increasing number of spectrin functions have been identified, the mechanism regulating these functions and the role of spectrin in some diseases are still unclear. We found that targeted genes regulated by tumor associated microRNA (miRNA) are also closely associated with the cytoskeletal signaling pathway [73,74]. Moreover, the levels of spectrin expression correlate negatively with those of certain miRNAs in patients with spectrin-related diseases. In silico analysis reveals that these miRNAs bind the 3'-UTR of spectrin mRNA, theoretically enabling negative regulation of gene expression; thus they could provide new avenues for studying the relationship between spectrins and their pathogenesis.

This work was supported by the National Natural Science Foundation of China (31000323, 31070672, 81250044), the Natural Science Foundation of Jiangsu Province (BK20131272), the Specialized Research Fund for the Doctoral Program of Higher Education of China (20100091120023), and the Fundamental Research Funds for the Central Universities (1095020823).

1 Marchesi V, Steers Jr E. Selective solubilization of a protein component of the red cell membrane. Science, 1968, 159: 203-204

2 Hartwig J H. Actin-binding proteins 1: Spectrin superfamily. Protein Profile, 1995, 2: 703-800

3 Naydenov N G, Ivanov A I. Spectrin-adducin membrane skeleton: A missing link between epithelial junctions and the actin cytoskeletion?
Bioarchitecture, 2011, 1: 186-191

4 Elgsaeter A, Stokke B T, Mikkelsen A, et al. The molecular basis of erythrocyte shape. Science, 1986, 234: 1217-1723

5 Stankewich M C, Cianci C D, Stabach P R, et al. Cell organization, growth, and neural and cardiac development require alphaII-spectrin. J Cell Sci, 2011, 124: 3956-3966

6 Cianci C D, Zhang Z, Pradhan D, et al. Brain and muscle express a unique alternative transcript of $\alpha$ II spectrin. Biochemistry, 1999, 38 : 15721-15730

7 Moon R T, McMahon A P. Generation of diversity in nonerythroid spectrins. Multiple polypeptides are predicted by sequence analysis of cDNAs encompassing the coding region of human nonerythroid $\alpha$-spectrin. J Biol Chem, 1990, 265: 4427-4433

8 Machnicka B, Grochowalska R, Boguslawska D M, et al. Spectrin-based skeleton as an actor in cell signaling. Cell Mol Life Sci, 2012, 69: 191-201

9 Dubreuil R R, Grushko T. Genetic studies of spectrin: New life for a ghost protein. Bioessays, 1998, 20: 875-878

10 Viel A. $\alpha$-Actinin and spectrin structures: An unfolding family story. FEBS Lett, 1999, 460: 391-394

11 Grum V L, Li D, MacDonald R I, et al. Structures of two repeats of spectrin suggest models of flexibility. Cell, 1999, 98: 523-535

12 Ipsaro J J, Harper S L, Messick T E, et al. Crystal structure and functional interpretation of the erythrocyte spectrin tetramerization domain complex. Blood, 2010, 115: 4843-4852

13 Brenner A K, Kieffer B, Travé G, et al. Thermal stability of chicken brain $\alpha$-spectrin repeat 17: A spectroscopic study. J Biomol NMR, 2012, 53: 71-83

14 Kusunoki H, Minasov G, MacDonald R I, et al. Independent movement, dimerization and stability of tandem repeats of chicken brain $\alpha$-spectrin. J Mol Biol, 2004, 344: 495-511

15 Li D, Harper S L, Tang H Y, et al. A comprehensive model of the spectrin divalent tetramer binding region deduced using homology modeling and chemical cross-linking of a mini-spectrin. J Biol Chem, 2010, 285: 29535-29545

16 Harper S L, Li D, Maksimova Y, et al. A fused $\alpha-\beta$ "mini-spectrin" mimics the intact erythrocyte spectrin head-to-head tetramer. J Biol Chem, 2010, 285: 11003-11012

17 Li D, Tang H Y, Speicher D W. A structural model of the erythrocyte spectrin heterodimer initiation site determined using homology modeling and chemical cross-linking. J Biol Chem, 2008, 283: 1553-1562

18 Li D, Harper S, David W. Initiation and propagation of spectrin heterodimer assembly involves distinct energetic processes. Biochemistry, 2007, 46: 10585-10594

19 Koshino I, Mohandas N, Takakuwa Y. Identification of a novel role for dematin in regulating red cell membrane function by modulating spectrin-actin interaction. J Biol Chem, 2012, 287: 35244-35250

20 Zhang P, Talluri S, Deng H, et al. Solution structure of the pleckstrin homology domain of Drosophila $\beta$ spectrin. Structure, 1995, 3: 1185-1195

21 Bennett V, Healy J. Organizing the fluid membrane bilayer: diseases linked to spectrin and ankyrin. Trends Mol Med, 2008, 14: 28-36

$22 \mathrm{Wu} \mathrm{S}$, Sangerman J, Li M, et al. Essential control of an endothelial cell ISOC by the spectrin membrane skeleton. J Cell Biol, 2001, 154: 1225-1234

23 Kizhatil K, Yoon W, Mohler P J, et al. Ankyrin-G and $\beta 2$-spectrin collaborate in biogenesis of lateral membrane of human bronchial epithelial cells. J Biol Chem, 2007, 282: 2029-2037

24 Devarajan P, Stabach P R, De Matteis M A, et al. Na, K-ATPase transport from endoplasmic reticulum to Golgi requires the Golgi spectrin-ankyrin G119 skeleton in Madin Darby canine kidney cells. Proc Natl Acad Sci USA, 1997, 94: 10711-10716

25 Cairo C W, Das R, Albohy A, et al. Dynamic regulation of CD45 lateral mobility by the spectrin-ankyrin cytoskeleton of T cells. J Biol Chem, 2010, 285: 11392-11401 
26 Zhang D Q, Wang Y P, Wang W H, et al. Interaction between protein 4.1R and spectrin heterodimers. Mol Med Report, 2011, 4: 651-654

27 Gimm J A, An X, Nunomura W, et al. Functional characterization of spectrin-actin-binding domains in 4.1 family of proteins. Biochemistry, 2002, 41: 7275-7282

28 An X, Debnath G, Guo X, et al. Identification and functional characterization of protein $4.1 \mathrm{R}$ and actin-binding sites in erythrocyte $\beta$ spectrin: Regulation of the interactions by phosphatidylinositol-4, 5-bisphosphate. Biochemistry, 2005, 44: 10681-10688

29 Becker P, Tse W, Lux S, et al. Beta spectrin kissimmee: A spectrin variant associated with autosomal dominant hereditary spherocytosis and defective binding to protein 4.1. J Clin Invest, 1993, 92: 612-616

30 Tse W T, Lux S E. Red blood cell membrane disorders. Br J Haematol, 1999,104: 2-13

31 Urwyler O, Cortinas-Elizondo F, Suter B. Drosophila sosie functions with beta(H)-spectrin and actin organizers in cell migration, epithelial morphogenesis and cortical stability. Biol Open, 2012, 1: 994-1005

32 Gushchina L V, Gabdulkhakov A G, Nikonov S V, et al. Highresolution crystal structure of spectrin $\mathrm{SH} 3$ domain fused with a proline-rich peptide. J Biomol Struct Dyn, 2011, 29: 485-495

33 Bialkowska K, Saido T C, Fox J E. SH3 domain of spectrin participates in the activation of Rac in specialized calpain-induced integrin signaling complexes. J Cell Sci, 2005, 118: 381-395

34 Collec E, Lecomte M C, El Nemer W, et al. Novel role for the $\mathrm{Lu} / \mathrm{BCAM}$-spectrin interaction in actin cytoskeleton reorganization. Biochem J, 2011, 436: 699-708

35 Benz P M, Blume C, Moebius J, et al. Cytoskeleton assembly at endothelial cell-cell contacts is regulated by alpha II-spectrin-VASP complexes. J Cell Biol, 2008, 180: 205-219

36 Rotter B, Bournier O, Nicolas G, et al. aII-Spectrin interacts with Tes and EVL, two actin-binding proteins located at cell contacts. Biochem J, 2005, 388: 631-638

37 Benz P M, Merkel C J, Offner K, et al. Mena/VASP and alphaII-spectrin complexes regulate cytoplasmic actin networks in cardiomyocytes and protect from conduction abnormalities and dilated cardiomyopathy. Cell Commun Signal, 2013, 11: 56

38 Mishra L, Cai T, Levine A, et al. Identification of elf1, a beta-spectrin, in early mouse liver development. Int J Dev Biol, 1998, 42: 221-224

39 Mishra L, Cai T, Yu P, et al. Elf3 encodes a novel 200-kD beta-spectrin: Role in liver development. Oncogene, 1999, 18: 353-364

40 Tang Y, Katuri V, Dillner A, et al. Disruption of transforming growth factor-beta signaling in ELF beta-spectrin-deficient mice. Science, 2003, 299: 574-577

41 Mishra B, Tang Y, Katuri V, et al. Loss of cooperative function of transforming growth factor-beta signaling proteins, smad3 with embryonic liver fodrin, a beta-spectrin, in primary biliary cirrhosis. Liver Int, 2004, 24: 637-645

42 Redman R S, Katuri V, Tang Y, et al. Orofacial and gastrointestinal hyperplasia and neoplasia in $\operatorname{smad}^{+/-}$and elf $^{+/-} /$smad $^{+/-}$mutant mice. J Oral Pathol Med, 2005, 34: 23-29

43 Stabach P R, Morrow J S. Identification and characterization of $\beta$ V spectrin, a mammalian ortholog of Drosophila $\beta_{\mathrm{H}}$ spectrin. J Biol Chem, 2000, 275: 21385-21395

44 Godi A, Santone I, Pertile P, et al. ADP ribosylation factor regulates spectrin binding to the Golgi complex. Proc Natl Acad Sci USA, 1998, 95: 8607-8612

45 de Matteis M A, Morrow J S. Spectrin tethers and mesh in the biosynthetic pathway. J Cell Sci, 2000, 113: 2331-2343

46 Salcedo-Sicilia L, Granell S, Jovic M, et al. betaIII spectrin regulates the structural integrity and the secretory protein transport of the Golgi complex. J Biol Chem, 2013, 288: 2157-2166

47 Sridharan D, Brown M, Lambert W C, et al. Nonerythroid alphaII spectrin is required for recruitment of FANCA and XPF to nuclear foci induced by DNA interstrand cross-links. J Cell Sci, 2003, 116: 823-835

48 McMahon L W, Zhang P, Sridharan D M, et al. Knockdown of al-
phaII spectrin in normal human cells by siRNA leads to chromosomal instability and decreased DNA interstrand cross-link repair. Biochem Biophys Res Commun, 2009, 381: 288-293

49 Zhang P, Herbig U, Coffman F, et al. Non-erythroid alpha spectrin prevents telomere dysfunction after DNA interstrand cross-link damage. Nucleic Acids Res, 2013, 41: 5321-5340

50 Machnicka B, Grochowalska R, Bogusławska D, et al. Spectrin-based skeleton as an actor in cell signaling. Cell Mol Life Sci, 2012, 69: 191-201

51 Grzybek M, Chorzalska A, Bok E, et al. Spectrin-phospholipid interactions: Existence of multiple kinds of binding sites? Chem Phys Lipids, 2006, 141: 133-141

52 Manno S, Takakuwa Y, Mohandas N. Identification of a functional role for lipid asymmetry in biological membranes: Phosphatidylserine-skeletal protein interactions modulate membrane stability. Proc Natl Acad Sci USA, 2002, 99: 1943-1948

53 Ray S, Chakrabarti A. Membrane interaction of erythroid spectrin: Surface-density-dependent high-affinity binding to phosphatidylethanolamine. Mol Membr Biol, 2004, 21: 93-100

54 Wolny M, Grzybek M, Bok E, et al. Key amino acid residues of ankyrin-sensitive phosphatidylethanolamine/phosphatidylcholinelipid binding site of $\beta \mathrm{I}$-spectrin. PLoS ONE, 2011, 6: e21538

55 Czogalla A, Grzymajło K, Jezierski A, et al. Phospholipid-induced structural changes to an erythroid $\beta$ spectrin ankyrin-dependent lipid-binding site. Biochim Biophys Acta, 2008, 1778: 2612-2620

56 Lecomte M C. Spectrins in human diseases. Cytoskeleton Hum Dis, 2012, 345-374

57 Harper S L, Sriswasdi S, Tang H Y, et al. The common hereditary elliptocytosis-associated alpha-spectrin L260P mutation perturbs erythrocyte membranes by stabilizing spectrin in the closed dimer conformation. Blood, 2013, 122: 3045-3053

58 Miraglia del Giudice E, Nobili B, Francese M, et al. Clinical and molecular evaluation of non-dominant hereditary spherocytosis. $\mathrm{Br} \mathrm{J}$ Haematol, 2001, 112: 42-47

59 Sakaguchi G, Orita S, Naito A, et al. A novel brain-specific isoform of beta spectrin: Isolation and its interaction with Munc13. Biochem Biophys Res Commun, 1998, 248: 846-851

60 Featherstone D E, Davis W S, Dubreuil R R, et al. Drosophila $\alpha$-and $\beta$-spectrin mutations disrupt presynaptic neurotransmitter release. $\mathrm{J}$ Neurosci, 2001, 21: 4215-4224

61 Ayala-Grosso C, Tam J, Roy S, et al. Caspase-3 cleaved spectrin colocalizes with neurofilament-immunoreactive neurons in Alzheimer's disease. Neuroscience, 2006, 141: 863-874

62 Jackson M, Song W, Liu M Y, et al. Modulation of the neuronal glutamate transporter EAAT4 by two interacting proteins. Nature, 2001, 410: 89-93

63 Waller K L, Nunomura W, An X, et al. Mature parasite-infected erythrocyte surface antigen (MESA) of Plasmodium falciparum binds to the $30-\mathrm{kDa}$ domain of protein 4.1 in malaria-infected red blood cells. Blood, 2003, 102: 1911-1914

64 Magowan C, Coppel R, Lau A, et al. Role of the Plasmodium falciparum mature-parasite-infected erythrocyte surface antigen (MESA/ PfEMP-2) in malarial infection of erythrocytes. Blood, 1995, 86: 3196-3204

65 Pei X, An X, Guo X, et al. Structural and functional studies of interaction between Plasmodium falciparum knob-associated histidine-rich protein (KAHRP) and erythrocyte spectrin. J Biol Chem, 2005, 280: 31166-31171

66 Herrera S, Rudin W, Herrera M, et al. A conserved region of the MSP-1 surface protein of Plasmodium falciparum contains a recognition sequence for erythrocyte spectrin. EMBO J, 1993, 12: 16071614

67 Ruetz T J, Lin A E, Guttman J A. Enterohaemorrhagic Escherichia coli requires the spectrin cytoskeleton for efficient attachment and pedestal formation on host cells. Microb Pathog, 2012, 52: 149-156

68 Samanta S, Dutta D, Ghoshal A, et al. Glycosylation of erythrocyte 
spectrin and its modification in visceral leishmaniasis. PLoS ONE, 2011, 6: e28169

69 Samanta S, Ghoshal A, Bhattacharya K, et al. Sialoglycosylation of $\mathrm{RBC}$ in visceral leishmaniasis leads to enhanced oxidative stress, calpain-induced fragmentation of spectrin and hemolysis. PLoS ONE, 2012, 7: e42361

70 Papal S, Cortese M, Legendre K, et al. The giant spectrin betaV couples the molecular motors to phototransduction and Usher syndrome type I proteins along their trafficking route. Hum Mol Genet, 2013, 22: 3773-3788

71 Witek M A, Fung L W. Quantitative studies of caspase-3 catalyzed
alphaII-spectrin breakdown. Brain Res, 2013, doi: 10.1016/ j.brainres.2013.08.010

72 Toporkiewicz M, Grzybek M, Meissner J, et al. Release of an 55 $\mathrm{kDa}$ fragment containing the actin-binding domain of beta-spectrin by caspase- 8 during FND-induced apoptosis depends on the presence of protein 4.1. Arch Biochem Biophys, 2013, 535: 205-213

73 Yang $H$, Zhang $H$, Zhu L, et al. Pathway analysis of cancer-associated microRNA targets. Int J Oncol, 2012, 41: 2213-2226

74 Zhang H, Yang H, Zhang R, et al. In-depth bioinformatic analysis of lung cancer-associated microRNA targets. Oncol Rep, 2013, 30: 2945-2956

Open Access This article is distributed under the terms of the Creative Commons Attribution License which permits any use, distribution, and reproduction in any medium, provided the original author(s) and source are credited. 